\title{
Gestión de datos geográficos en bibliotecas universitarias españolas: Estado de la cuestión
}

\author{
Estefania Aguilar-Moreno*, Carlos Granell-Canut* \\ *Institute of New Imaging Technologies. Universitat Jaume I de Castellón (España). \\ Correo-e: eaguilar@uji.es, carlos.granell@uji.es
}

Recibido: 10-06-2014; 2a versión: 27-10-2014; Aceptado: 07-11-2014.

Cómo citar este artículo/Citation: Aguilar-Moreno, E.; Granell-Canut, C. (2015). Gestión de datos geográficos en bibliotecas universitarias españolas: Estado de la cuestión. Revista Española de Documentación Científica, 38(2): e085. doi: http://dx.doi. org/10.3989/redc.2015.2.1193

Resumen: Este trabajo presenta los resultados de una encuesta sobre la gestión de datos geográficos digitales en bibliotecas universitarias españolas. El objetivo de la investigación es averiguar en qué estado se encuentran las bibliotecas universitarias españolas en relación con la gestión de datos geográficos y cuáles pueden ser los factores limitantes para su desarrollo. Los resultados reflejan la percepción de las bibliotecas universitarias sobre cuál podría ser su rol dentro de la gestión de datos geográficos en el entorno universitario.

Palabras clave: Encuesta; bibliotecas universitarias; datos geográficos; servicios geográficos en bibliotecas.

Geographic data management in Spanish university libraries: state of the art

Abstract: This paper presents the results of a survey on the management of digital geographic data in Spanish academic libraries. The objective of the research is to discover the state of Spanish university libraries in relation to the management of geographic data, as well as factors that might hinder it. The results reflect the perception of university libraries on their role in managing geographic data in the academic environment.

Keywords: Survey; university libraries; geographic data; geographic services in libraries.

Copyright: (c) 2015 CSIC. Este es un artículo de acceso abierto distribuido bajo los términos de la licencia Creative Commons Attribution-Non Commercial (by-nc) Spain 3.0. 


\section{INTRODUCCIÓN}

En la actualidad los mapas son mucho más que una representación bidimensional de puntos. Son productos digitales generados dinámicamente como resultado de la superposición de diversas capas de información a gusto del usuario. Cada una de estas capas se compone de un conjunto de datos geográficos, es decir, datos que llevan asociada una posición geográfica que, al estar conectados con otros datos y puestos sobre un mapa base, dan a la información presentada mayor sentido (Aguilar-Moreno y Granell-Canut, 2013). Los Sistemas de Información Geográfica (SIG) son herramientas que facilitan la tarea de combinar diferentes capas de datos geográficos para la elaboración de mapas, los cuales pueden servir como herramienta de decisión y visualización de la información.

Los datos geográficos destacan especialmente por su horizontalidad e interdisciplinariedad (Scaramozzino y otros, 2014), y por la diversidad de materias en los que se pueden emplear (Kinikin y Hench, 2005a), tanto en disciplinas científicotécnicas (Conway y otros, 2013), como en ciencias sociales o humanas (Bosque González y otros, 2012). Vardakosta y Kapidakis (2012) hacen notar la existencia de una gran variedad de datos geográficos, en cuanto a su tipología, formato y materia. Debido a la facilidad de generación y captación de los datos geográficos (Goldberg y otros, 2014), estos se generan o se utilizan comúnmente en proyectos de investigación académica de diversa índole. De hecho, los datos geográficos y las tecnologías geoespaciales han trascendido en la actualidad sus ámbitos originarios de uso (Geografía, Topografía, etc.) para ser empleados en muchas otras disciplinas científicas, tanto en Ciencia e Ingeniería, como en Ciencias Sociales y Humanidades (Balschke y Merschdorf, 2014), estando en consecuencia presentes en la mayoría de los departamentos universitarios.

Esta gran difusión - con diversos grados de profundidad - ha propiciado que, muchas bibliotecas universitarias (principalmente en el ámbito anglosajón), consideren los datos geográficos como una colección más a gestionar (Goldberg y otros, 2014). Para llevar a cabo estas tareas, las universidades requieren un perfil profesional mixto, todavía algo impreciso, denominado geo-librarian o GIL (Geographic Information Librarian) (Weimer y Reehling, 2006). En el contexto de este trabajo lo denominaremos "geobibliotecario" (Granell-Canut y Aguilar-Moreno, 2013).

Observando los grandes avances llevados a cabo por las bibliotecas universitarias estadounidenses desde los años 90 y el nivel de profesionalización que han llegado a alcanzar en relativamente poco tiempo (20 años), el presente trabajo pretende explorar que está sucediendo en el contexto académico español. Haciendo una revisión bibliográfica, prácticamente no se encuentra ninguna publicación española sobre la temática, únicamente
Pérez Pulido (1999) y Lorite (2011) dan algunos apuntes al respecto. Esto nos lleva a pensar que, hasta el momento, los datos geoespaciales o geográficos no están siendo considerados como parte de la colección a gestionar dentro de la biblioteca universitaria española, al tiempo que el perfil del geobibliotecario no se encuentra recogido específicamente dentro de sus plantillas.

El presente trabajo viene motivado por la falta de investigaciones y estudios referentes a la gestión de datos geográficos desde las bibliotecas universitarias españolas. Por lo tanto, el propósito general de este artículo es iniciar una discusión sobre cuál es o podría ser el papel de las bibliotecas universitarias españolas en cuanto a la gestión de datos geográficos y qué beneficios podría reportar su gestión a las bibliotecas. Este objetivo general se plasma en los siguientes objetivos concretos:

- Averiguar si existen políticas activas en cuanto a la gestión de datos geográficos.

- Detectar los principales impedimentos o carencias (por ejemplo falta de recursos económicos y humanos, software, hardware, licencias de acceso a datos, repositorios de datos, etc.) que afectan al desarrollo y puesta en marcha de servicios específicos para la gestión de datos geográficos en el contexto universitario español.

- Indicar el grado de colaboración entre las bibliotecas universitarias y el resto de departamentos e institutos universitarios en materia de gestión y acceso de datos geográficos. La falta de conocimiento interno entre los organismos de una universidad puede acarrear una falta de colaboración entre los generadores de datos geográficos y las bibliotecas.

- Analizar el perfil profesional actual encargado de la gestión de datos geográficos.

- Valorar la visión que tienen las bibliotecas universitarias españolas sobre las competencias deseables de un perfil de geobibliotecario.

\section{METODOLOGÍA}

El presente trabajo parte de la misma base que motivó los trabajos de Vardakosta y Kapidakis (2011a) y Kelly (2013). Ambos trabajos detectaron la deficiente implantación de servicios relacionados con los datos geográficos en bibliotecas académicas griegas y sudafricanas, respectivamente. Para verificar el nivel de implementación de estos servicios en las bibliotecas, Vardakosta y Kapidakis (2011a) estudiaron las páginas web de las bibliotecas de aquellas universidades en las que existía un departamento de Geografía, Geología, Topología, Ciencias de la Tierra o Medioambiente. Por su parte, Kelly (2013) se centró en el estudio de los sitios web de las bibliotecas universitarias mejor posicionadas en el Academic Ranking of World 
Universities (http://www.shanghairanking.com/). Vardakostas y Kapidakis (2011b) concluyeron que solo el $30 \%$ de las bibliotecas estudiadas a nivel mundial, de entre un estudio que comprendió 160 universidades, ofrecían servicios relacionados con datos geográficos. Como hemos comentado anteriormente, la horizontalidad de los datos geográficos nos obliga a aproximarnos a la cuestión desde una perspectiva más amplia. El presente estudio no se limita al estudio de las universidades con departamentos tradicionalmente asociados a la recolección, gestión y explotación de datos geográficos, sino que considera que cualquiera departamento puede generar o necesitar este tipo de datos. A este respecto en este trabajo consideramos que, dentro del sistema universitario español, no siempre los departamentos declaran abiertamente su orientación al uso de datos geográficos y tecnologías geoespaciales, sino que esta información subyace en el desarrollo de sus investigaciones.

En el contexto español, el rol de la biblioteca como centro de servicios de datos geográficos parece que todavía no se ha desarrollado (Granell-Canut y Aguilar-Moreno, 2013), por lo tanto carece de sentido analizar las webs de las bibliotecas en la búsqueda de la oferta de servicios de este tipo tal como hicieron Vardakosta y Kapidakis (2011a) y Kelly (2013). Por lo tanto, el estudio planteó la premisa inicial de que las bibliotecas universitarias españolas no están ofreciendo servicios en relación con la gestión de datos geográficos o que se encontrarían en un estado embrionario y por lo tanto aún no serían visibles a través de los portales web bibliotecarios.

Otros autores hacen uso de encuestas para recoger información sobre el nivel de implantación de servicios de datos geográficos y SIG en bibliotecas universitarias o científicas, con el fin de identificar qué servicios se ofertan. En la mayoría de la literatura analizada se estudian: los servicios ofrecidos, personal dedicado, infraestructuras, y presupuesto dedicado (ARL, 1999; Kinikin y Hench, 2005a; Kinikin y Hench, 2005b; Cullingworth, 2005). El trabajo de Gabaldón y Repplinger (2006) diseña una encuesta básica sobre el uso de software SIG en relación con otras variables, poco relevante para nuestro estudio. Por su parte, Cullingworth (2005) reúne las cuestiones planteadas en otras encuestas, distinguiéndose de ellas al estudiar el nivel de catalogación empleado (datasets, ficheros, series). Este es el único trabajo encontrado que se preocupa del estudio del uso de datos geográficos generados por la propia universidad, punto importante a la hora de estudiar el nivel de colaboración entre la comunidad SIG y biblioteca.

Todos los trabajos anteriores parten de la base de que las bibliotecas ya disponen de servicios de datos geográficos y SIG, por lo tanto solo alguna cuestión general de esas encuestas podía ayudarnos en la definición de nuestra encuesta en el contexto del presente estudio. Tras analizar los formu- larios de encuesta publicados hasta el momento, se definió un modelo específico para descubrir en qué estado de desarrollo se encuentran las bibliotecas universitarias españolas con relación a la gestión de datos geográficos y sus planes de futuro con respecto a la gestión de datos geográficos. Se procedió pues a elaborar una encuesta dirigida a las bibliotecas universitarias españolas adscritas a REBIUN ${ }^{1}$. El universo fueron las bibliotecas universitarias españolas $(\mathrm{N}=73)$.

La encuesta ${ }^{2}$ se estructuró en cuatro bloques: Políticas y materiales; Colaboración y acceso a datos geográficos; Perfiles específicos; y Competencias específicas. En función de las respuestas dadas por cada biblioteca participante el formulario de encuesta guiaba a los participantes hacia la siguiente pregunta, de modo que pudieran crearse recorridos diferentes al inicio de cada bloque. El bloque 1 diferenciaba entre las bibliotecas que gestionaban recursos geográficos y las que no para profundizar en caso positivo en los recursos utilizados. El bloque 2 preguntaba si las bibliotecas tenían constancia o no de que en la universidad otros departamentos trabajaran con datos geográficos. El bloque 3 formulaba diferentes preguntas a las bibliotecas que disponían o no de personal específico para desarrollar estas tareas. Finalmente, el bloque 4 valoraba la opinión de las bibliotecas encuestadas sobre las competencias profesionales que se deberían poseer para llevar a cabo la tarea de gestión de datos geográficos.

El formulario de la encuesta se colgó en internet haciendo uso de una herramienta de creación de encuestas online de Google. Debido al carácter estratégico que pueda suponer el desarrollo de esta nueva faceta por parte de la biblioteca, la encuesta se dirigió a los directores de las bibliotecas, siendo estos libres de redirigir su participación en el estudio a otras personas más idóneas dentro de la biblioteca.

Se envió un email en el mes de enero 2014 invitando a las bibliotecas a participar. En febrero 2014 se envió un recordatorio a aquellas bibliotecas que no hubieran respondido a la encuesta, enfatizando nuestro interés en la colaboración de bibliotecas sin política sobre datos geográficos, ya que alguna biblioteca había indicado que no creía interesante su participación puesto que no contemplaban los datos geográficos dentro de sus planes de desarrollo. En mayo 2014 se procedió al análisis ${ }^{3}$ de los resultados.

\section{RESULTADOS Y DISCUSIÓN}

La encuesta fue respondida por 28 bibliotecas universitarias repartidas por toda la geografía nacional $^{2}$. La gran mayoría de universidades participantes fueron públicas (24) frente a 4 universidades privadas. Cada una de las secciones siguientes trata un bloque específico de la encuesta, a excepción del bloque 1 que se divide en dos secciones (3.1 y 3.2). 


\subsection{Políticas activas en materia de datos geográficos}

El primer dato relevante es que sólo 6 universidades declararon que contemplan la gestión de datos geográficos (tabla I), enmarcando su gestión dentro de la política de gestión de la colección. Según estos datos, aproximadamente una de cada cinco bibliotecas universitarias españolas tiene en cuenta los datos geográficos en sus políticas, lo cual no estaría tan lejos del $30 \%$ a nivel mundial expuesto por Vardakostas y Kapidakis (2011b). No obstante, este porcentaje es engañoso y presumiblemente a la baja ya que la encuesta tiene en cuenta escasamente el $40 \%$ del total de bibliotecas universitarias españolas. En el reciente trabajo de Herrera-Morillas (2013) sobre gestión de colecciones en bibliotecas universitarias españolas, el autor concluye que únicamente un $14 \%$ de las 71 bibliotecas universitarias analizadas declararon disponer de un plan de gestión y/o desarrollo de colecciones. En este contexto, no sorprendería en absoluto que el porcentaje real sobre el total de las bibliotecas que prestan atención a la gestión de datos geográficos fuera bastante menor.

Lorite (2011) posiciona a las bibliotecas universitarias con un mayor número de documentos cartográficos, como las más interesadas, a priori, en los datos geográficos. Este autor identifica seis universidades que acopian el $50 \%$ de las colecciones cartográficas de las bibliotecas universitarias españolas: Universidad Complutense de Madrid (UCM), Universitat Autónoma de Barcelona (UAB), Universidad Autónoma de Madrid (UAM), Universidad Politécnica de Madrid (UPM), Universitat de Barcelona (UB) y Universitat de València (UVEG). Comparando los datos que ofrece Lorite, con los obtenidos en el presente trabajo, solo UCM y UAB estarían evolucionando hacia la gestión de datos geográficos, mientras que UPM, UB y UVEG parecen no seguir este camino de acuerdo a las respuestas en nuestra encuesta.

Frente a esto cabe sin embargo puntualizar que la cobertura y naturaleza de los recursos cartográficos es distinta con respecto a los datos geográficos. Los recursos cartográficos forman parte de los datos geográficos pero no son los únicos. La noción de dato geográfico va mucho más allá del recurso cartográfico en cuanto que incide en el dinamismo de los propios datos (no tienen por qué ser únicamente recursos estáticos como sucede por ejemplo con los mapas cartográficos antiguos) los cuales por definición contienen una referencia explícita a una localización determinada (punto, área, etc.), son voluminosos, varían en escala y pueden encadenar numerosos procesos y algoritmos (Ariza López y otros, 2012). Los datos resultados de tareas de investigación son un ejemplo de datos geográficos que no siempre vienen representados en forma de mapa - como sucede a menudo con los recursos cartográficos - sino en forma de dataset que pueden ser utilizados en un producto o servicio final como un mapa o como datos de entrada para otras tareas o procesos de investigación.

Observando la tabla I, cabe destacar también que la UA y la UDG van más allá de la mera gestión de colecciones y consideran la gestión de los datos geográficos como parte de la política en gestión de datos de investigación y datos abiertos. Desafortunadamente, se trata aún de una tendencia prácticamente testimonial entre las bibliotecas universitarias españolas, a diferencia de otras bibliotecas en el ámbito internacional, principalmente americanas, donde sí incluyen los datos geográficos como parte de los datos a conservar siguiendo las recomendaciones sobre datos abiertos y gestión de los datos de investigación (Goldberg y otros, 2014).

\subsection{Recursos materiales disponibles}

El coste de mantenimiento de los datos geográficos, sea desde la perspectiva de las infraestructuras (hardware y/o software) necesarias, como del personal especializado, son indiscutiblemente una carga difícil de llevar que limita, en gran medida, la oferta de servicios geográficos (Kinikin y Hench, 2005b). Cullingworth (2005) apunta, desde el contexto canadiense, que la accesibilidad a los datos geográficos, en cuanto a costes y problemas de copyright, también puede influir enormemente en la decisión de gestionar servicios geográficos des-

Tabla I. Universidades que contemplan planes de gestión de datos geográficos (Bloque 1)

\begin{tabular}{|l|c|c|c|}
\hline Universidad & $\begin{array}{c}\text { Gestión de } \\
\text { colecciones }\end{array}$ & $\begin{array}{c}\text { Gestión de datos } \\
\text { de investigación }\end{array}$ & $\begin{array}{c}\text { Política de Datos } \\
\text { Abiertos }\end{array}$ \\
\hline Universidad Complutense de Madrid (UCM) & Sí & No & No \\
\hline Universidad de Granada (UGR) & Sí & No & No \\
\hline Universidad de Murcia (UM) & Sí & No & No \\
\hline Universitat Autónoma de Barcelona (UAB) & Sí & No & Sí \\
\hline Universitat d'Alacant (UA ) & Sí & Sí & Sí \\
\hline Universitat de Girona (UDG) & Sí & Sí & \\
\hline
\end{tabular}


de la biblioteca. Sin embargo, según vemos en la figura 1, que muestra los diferentes motivos ente las bibliotecas encuestadas que no contemplan planes de gestión de datos geográficos $(\mathrm{N}=22)$, las bibliotecas en general, no consideran ni la falta de recursos económicos ni humanos como los principales motivos de la falta de servicios de datos geográficos. La inmensa mayoría coincide en que no existe la necesidad ni la prioridad para llevar a cabo servicios de gestión de datos geográficos. Incluso en cinco ocasiones se menciona a otras instituciones o departamentos como responsables de la gestión de datos geográficos. Estos datos podrían corroborarse con el estudio llevado a cabo por Ariza López y otros (2012), en donde algunas universidades señalan a los departamentos de Geografía como gestores de datos geográficos, permaneciendo las bibliotecas universitarias ajenas a las prácticas de curación y preservación de este tipo de datos.

Curiosamente en el caso español, a diferencia de lo que arrojan los datos de estudios de bibliotecas universitarias estadounidenses (Florance, 2006), el principal obstáculo no se debe a la falta de recursos humanos o económicos, sino que simplemente no se percibe la gestión de los datos geográficos como un servicio propio que deban desempeñar las bibliotecas universitarias. Parte del problema, en nuestra opinión, se debe a una falta de conocimiento con respecto al papel que realmente juegan los datos geográficos dentro de las políticas de datos de investigación y de datos abiertos (Hernández Pérez y García Moreno, 2013). Como hemos visto en la tabla I, los datos geográficos se identifican mayoritariamente como una colección más, y en raras ocasiones como parte de los datos de investigación y como datos abiertos. Sin embargo, en portales pioneros en datos abiertos como data. gov y data.gov.uk, los datos geográficos son el tipo de datos mayoritario con diferencia con respecto al total de los datos recogidos en esos portales. Si se realiza una búsqueda con más atención, los datos pertenecientes a otras categorías como salud y educación también incluyen a menudo referencias geográficas, poniendo de manifiesto la transversalidad de la dimensión geográfica en multitud de temáticas y dominios distintos.

Figura 1. Motivos de la falta de planes de gestión de los datos geográficos en las bibliotecas, $\mathrm{N}=22$ (Bloque 1 )

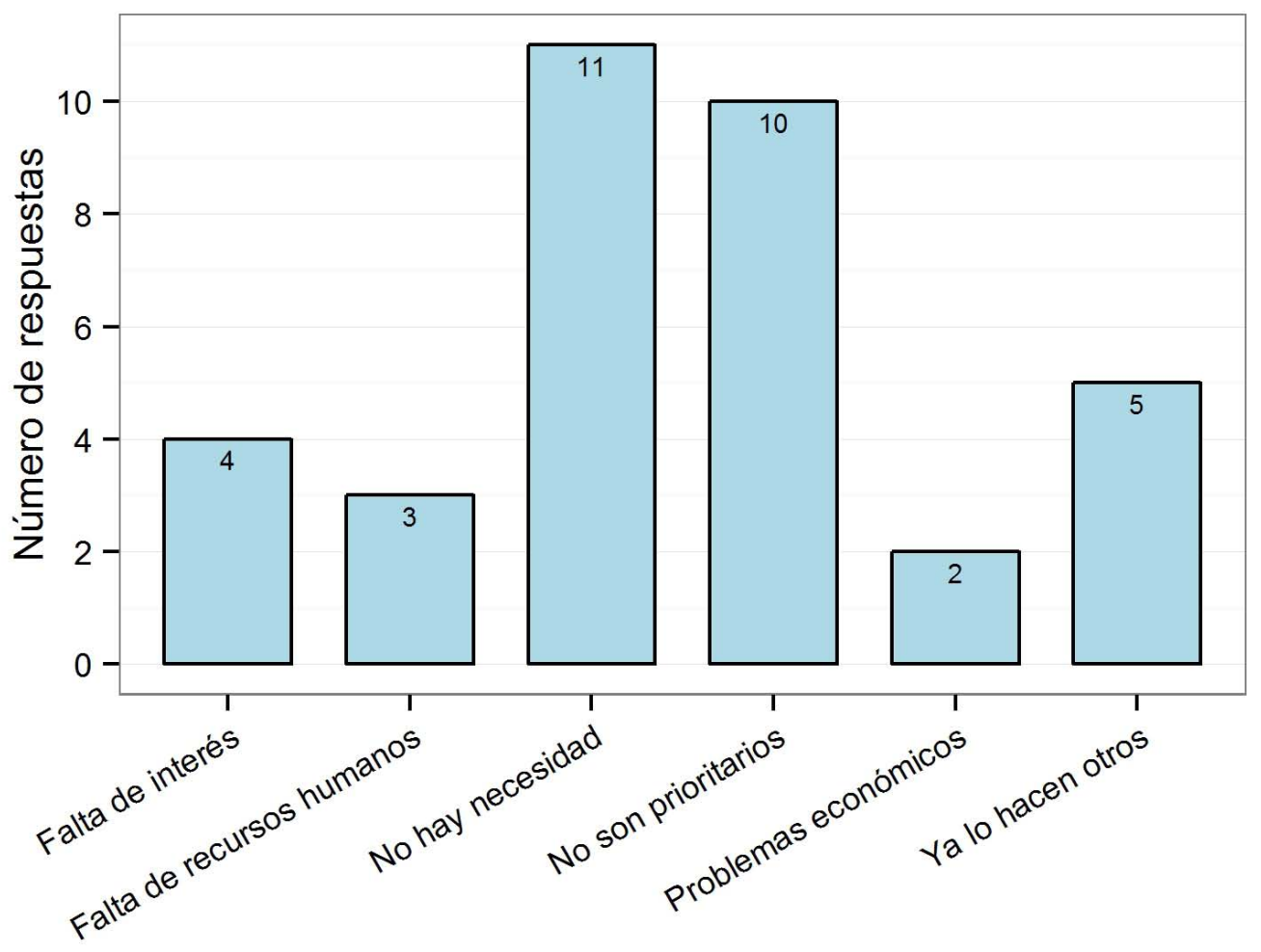

La suma es superior a $\mathrm{N}$ por la selección múltiple de las respuestas. 
En lo que respecta a las bibliotecas españolas que gestionan datos geográficos, solo UA (Alicante) y UM (Murcia) disponen de un repositorio específico para datos geográficos; UGR (Granada) y UAB (Autónoma de Barcelona) mantienen los datos en repositorios de carácter general. Por su parte UdG (Girona) utiliza el repositorio de la cartoteca y UCM (Complutense) aún no ha definido un plan para su gestión. La UA y UAB han desarrollado un repositorio en colaboración con otros departamentos universitarios, mientras que en los casos de UGR y UM ha sido la propia biblioteca la responsable del desarrollo del repositorio. En definitiva, el desarrollo de estos repositorios viene siendo desigual y no se aprecia, en función de los datos obtenidos, una tendencia clara en cuanto a la estrategia para la puesta en marcha de los repositorios de soporte para la gestión de datos geográficos.

\subsection{Colaboración y acceso a datos geográficos}

La colaboración entre diferentes departamentos que utilizan datos geográficos y/o SIG es fundamental, no sólo para tener perspectivas de éxito a la hora de ofertar este tipo de servicios desde la biblioteca, como reconoce Jantz (1997), sino para el desarrollo y puesta en funcionamiento del servicio en sí mismo (Guan y otros, 2011). La biblioteca puede hacer uso de su posición neutral para coordinar y definir el servicio de datos geográficos y posicionarse como punto de referencia entre los departamentos y servicios académicos que hacen uso de datos geográficos. Varios autores (Cline y Adler, 1995; Guan y otros, 2011) describen experiencias de cómo la biblioteca - como centro recolector de datos geográficos generados dentro de la propia universidad - propicia sinergias y colaboraciones más intensas y duraderas con otros departamentos que trabajan con datos geográficos.

En cuanto a los resultados que se desprenden del presente estudio sobre el acceso a datos geográficos y colaboración con otros departamentos por parte de las bibliotecas universitarias españolas, podemos matizar los siguientes aspectos.

En primer lugar, 16 de las 28 bibliotecas encuestadas son conscientes de que otros departamentos de la propia universidad generan o usan datos geográficos. Sin embargo, no se llegan a establecer colaboraciones con esos departamentos. Únicamente tres bibliotecas declaran que tienen establecido algún tipo de colaboración en la que la biblioteca participa en iniciativas conjuntas en gestión de datos geográficos. En particular, la UGR actúa como receptor de datos geográficos de otros departamentos, la UA desarrolla repositorios de gestión de datos geográficos, y la UDG participa en el desarrollo de la cartoteca. En definitiva, las bibliotecas universitarias presentan un bajo nivel de interacción con la comunidad SIG presente en sus universidades, en la misma línea de un comentario que Ariza López y otros (2012) recogen en un reciente trabajo donde concluyen que "en el ámbito universitario existe una desconexión total entre los departamentos que generan información geográfica y las bibliotecas y archivos de estas instituciones".

¿A qué puede deberse la escasa colaboración entre bibliotecas y departamentos con respeto a los datos geográficos? Esta observación se explica parcialmente con los resultados presentados en la figura 1, donde la percepción general de las bibliotecas universitarias españolas es que la gestión de los datos geográficos no se encuentra entra sus prioridades. No parece existir pues ningún estímulo, a corto o medio plazo, para establecer contactos y sinergias con otros departamentos e institutos universitarios en materia de gestión de datos geográficos. Como apuntábamos anteriormente, la falta de necesidad pueda deberse a que las bibliotecas no identifican los datos geográficos como parte de la política de gestión de datos de investigación, política cada vez más importante dentro del mundo universitario. Todos estos factores en conjunto propician que la colaboración entre biblioteca y departamentos no despegue, acentuando además tanto la falta de acceso a datos geográficos que se generan o utilizan en el seno de la propia universidad, como la reutilización de los mismos.

Sin embargo, como Goldberg y otros (2014) reconocen, la gestión de datos geográficos no sólo forma parte de la política de gestión de datos de investigación, sino que puede llegar a tener un papel estratégico dentro del seno de la propia universidad. Por ejemplo, recientemente se habla mucho de las iniciativas de Ciudades Inteligentes. La principal justificación de este tipo de proyectos es la eficiencia energética y el ahorro de costes en la gestión de los servicios públicos a los ciudadanos. La puesta en marcha de aplicaciones y servicios para afrontar la eficiencia energética y la movilidad, por citar dos ejemplos característicos en las Ciudades Inteligentes, conlleva necesariamente tener en cuenta la dimensión espacial de la propia ciudad y, en consecuencia, los datos geográficos son de vital importancia. Esta misma consideración se aplica a los Campus Inteligentes, que son proyectos de Ciudades Inteligentes a pequeña escala pero que se desarrollan en un entorno real, lo cual los hace idóneos como laboratorio de pruebas de aplicaciones y servicios que posteriormente puedan transferirse al contexto de las Ciudades Inteligentes (Gould, 2013). Por ejemplo, la movilidad de los estudiantes, profesores, personal de apoyo y visitantes a través del campus o en el interior de los edificios, los planes de evaluación de seguridad, la definición y mantenimiento de los planes de transporte público, la mejora en la gestión de estacionamiento de coches en el propio campus, etc. Todos estos ejemplos de servicios y aplicaciones tienen como 
denominador común la dimensión geográfica y espacial (Johnson y Phoenix, 2003). En este contexto, la gestión de datos geográficos a nivel de campus universitario, que dé soporte a servicios y aplicaciones para la universidad, se convierte en una pieza clave para el éxito de los Campus Inteligentes. Y es aquí donde las bibliotecas universitarias pueden y deben jugar un papel primordial en la gestión de las colecciones de datos geográficos, manteniendo estrechas colaboraciones con los demás actores (departamentos, rectorado, servicios de mantenimiento, etc.) para aunar conocimientos en proyectos estratégicos como el ejemplo de los Campus Inteligentes.

Por último, nos pareció interesante conocer qué tipo de colaboración sería deseable establecer desde la biblioteca con otros departamentos en materia de datos geográficos, aunque actualmente no exista ningún lazo de colaboración (figura 2). De las 16 bibliotecas que afirmaron conocer que otros departamentos realizan labores de gestión de datos geográficos, la mayoría se inclina por una colaboración que implicase el desarrollo de repositorios de gestión de datos geográficos, seguido de cerca por la participación conjunta entre la biblioteca universitaria y otros departamentos en iniciativas sobre el uso y explotación de datos geográficos. Curiosamente, la opción de convertir la biblioteca universitaria en un centro receptor de datos geográficos no parece un tipo de colaboración que interese a las bibliotecas universitarias encuestadas, a pesar de que este tipo de colaboración ayuda inicialmente a afianzar relaciones con otros departamentos tal como lo demostraron los casos de éxito de universidades académicas americanas comentados anteriormente. Scaramorzzino y otros (2014) ven a la biblioteca principalmente como centro receptor de datos geográficos, de carácter neutral, capaz de ejercer funciones puente entre diferentes perfiles dentro de la universidad. Este tipo de colaboración inicial actúa como un trampolín para establecer lazos más duraderos entre bibliotecas y departamentos universitarios y abrir así las puertas a futuras colaboraciones en proyectos conjuntos.

Figura 2. Colaboración deseable entre las bibliotecas y otros departamentos de la universidad, $\mathrm{N}=16$ (Bloque 2)

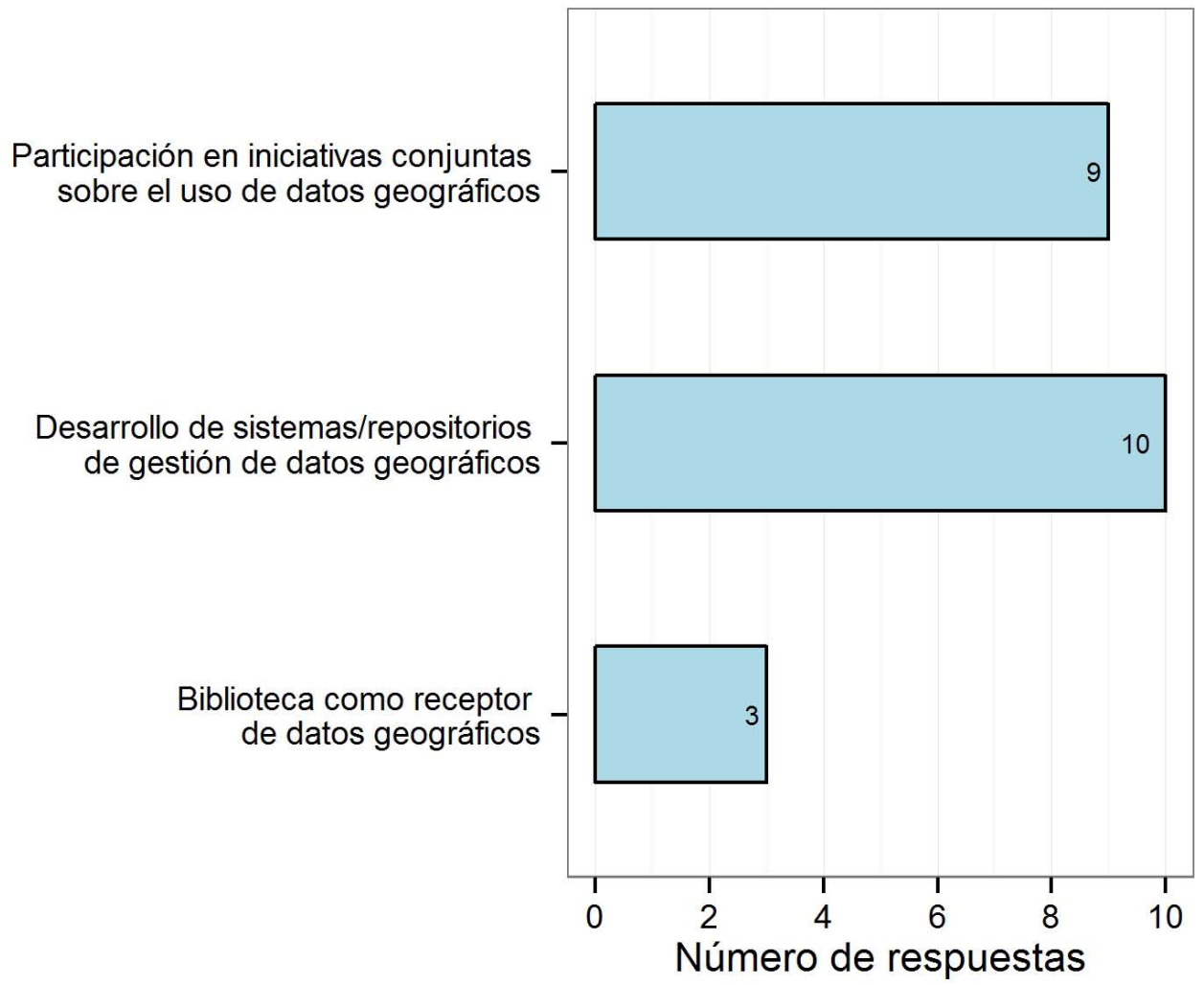

La suma es superior a $\mathrm{N}$ por la selección múltiple de las respuestas. 


\subsection{Recursos humanos disponibles en gestión de datos geográficos}

Los resultados que arroja la encuesta no son muy esperanzadores en cuanto a la existencia de perfiles específicos para la gestión de datos geográficos en las bibliotecas universitarias españolas. Del total de las bibliotecas universitarias encuestadas, 24 no dispone de personal, bien propio o externo, especializado en la gestión de datos geográficos. De las pocas que sí lo tienen (UAB, UVEG, UA, UDG), se trata de personal propio de la plantilla al que se le ha proporcionado formación específica. Es decir, el perfil actual es un bibliotecario de formación especializado en recursos cartográficos.

La falta de perfiles específicos y vías formativas especializadas para los bibliotecarios en estas temáticas, afirma Martindale (2004), también dificultan la oferta y puesta en marcha de este tipo de servicios. Los programas académicos españoles en Información y Documentación, a diferencia de los estadounidenses, como por ejemplo describe Dodsworth (2009), carecen de formación específica en gestión de datos geográficos y herramientas SIG dentro del contexto bibliotecario. En ausencia de un programa curricular específico, estas competencias pueden desarrollarse: (a) dando formación especializada a los bibliotecarios en plantilla, (b) logrando la participación conjunta de varios perfiles bibliotecarios expertos (bases de datos, metadatos, desarrollo de la colección, etc.) (Longstreth, 1995), o (c) mediante la creación de equipos multi- disciplinares de trabajo con participación de personal bibliotecario y expertos en datos geográficos y herramientas SIG (Cline y Adler, 1995). Longstreth (1995) identifica como más adecuada la participación de consultores externos en las fases iniciales del desarrollo y planificación de un servicio de datos geográficos, mientras que el personal interno es más efectivo a la hora de mantener el servicio por el conocimiento que tienen de la institución en la que trabajan. Por otra parte, de acuerdo a las experiencias que reflejan Cline y Adler (1995) y Guan y otros (2011), en el largo plazo, es importante contar con una persona del ámbito bibliotecario, pero con conocimientos amplios en datos geográficos y herramientas SIG, que gestione y coordine el servicio.

A raíz de un trabajo anterior, donde se nos planteó la duda de si una oferta laboral específica para la gestión de datos geográficos, tal como sucede en el contexto norteamericano, podría ser cubierta adecuadamente en el contexto español (GranellCanut y Aguilar-Moreno, 2013), creímos interesante conocer la visión de las bibliotecas en cuanto al perfil idóneo para la gestión de datos geográficos (figura 3). Para la definición de los posibles perfiles de la encuesta, partimos de la premisa establecida en el Informe MAGERT (2008, actualizado en 2012) sobre competencias base para geobibliotecarios, donde se establecen tres tipos de perfiles: bibliotecario de mapas, bibliotecario SIG y bibliotecario de catalogación y metadatos.

Figura 3. Perfil profesional idóneo para la gestión de datos geográficos, $N=28$ (Bloque 3)

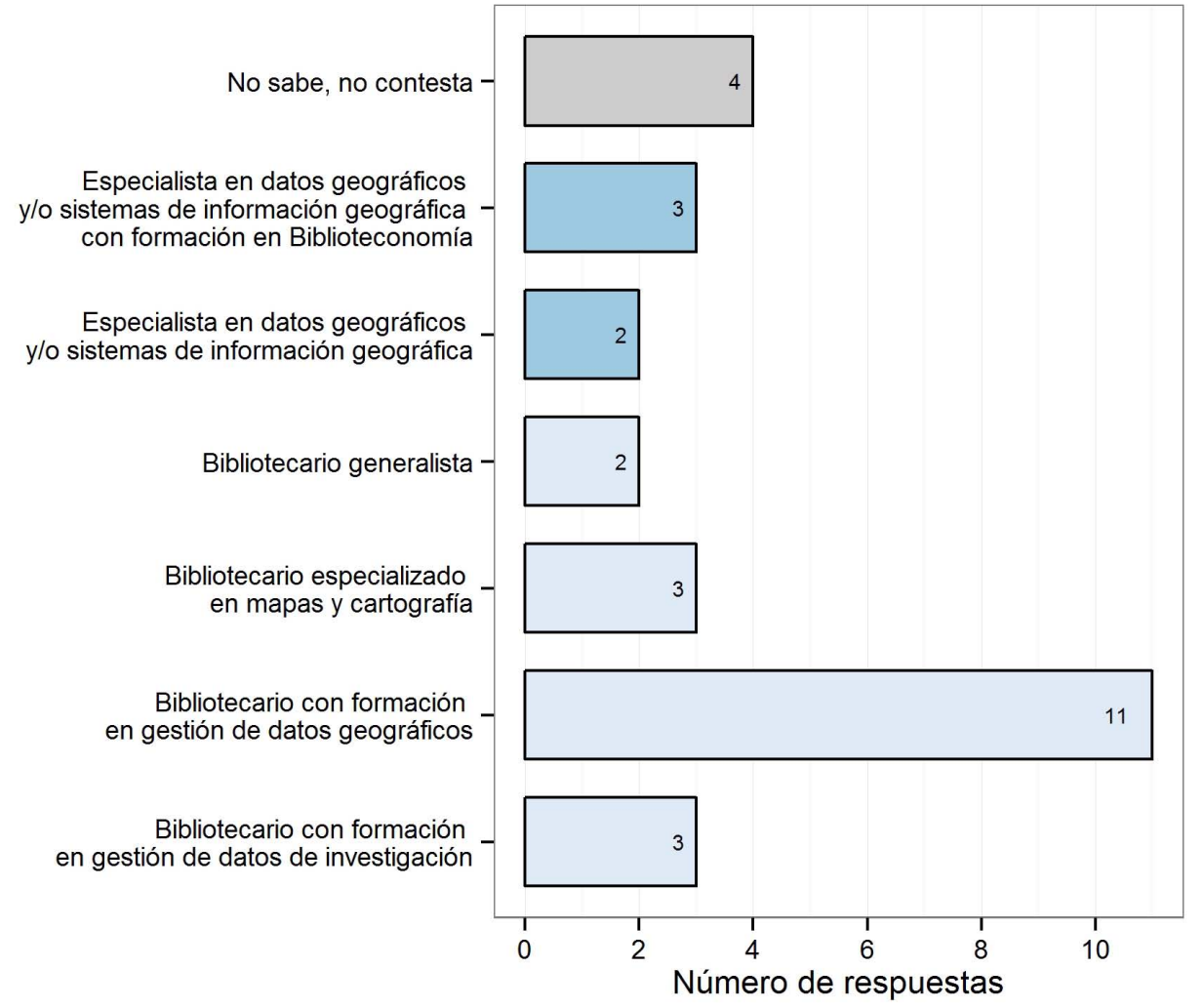


El primer perfil que definimos se correspondería con un candidato con formación principal en Información y Documentación, complementada con conocimientos en datos geográficos y sus variantes (barras coloreadas en azul claro en la figura 3). El segundo perfil es justo el opuesto, un perfil que prioriza la especialidad en datos geográficos con formación adicional en biblioteconomía (las dos barras coloreadas en azul oscuro en la figura 3), lo que el informe MAGERT (2008) denomina bibliotecario SIG. La gran mayoría de las respuestas se inclinan por el primer perfil, con formación de base en Biblioteconomía y con conocimientos específicos en datos geográficos. Esta opción es, de hecho, el perfil más demandado en ofertas de empleo de universidades americanas (Granell-Canut y Aguilar-Moreno, 2013). Sin embargo, el perfil ideal no coincide con la realidad actual de las bibliotecas universitarias que sí disponen de un plan de gestión de datos geográficos, en las cuales prima un perfil profesional focalizado en mapas y cartografía. Cabe recordar que la gestión de datos geográficos engloba mucho más que cartografía y mapas, lo cual permitiría a las bibliotecas establecer nuevos servicios y colaboraciones con una mayor variedad de usuarios y disciplinas científicas dentro de la propia universidad.

\subsection{Competencias deseables de un geobibliotecario}

La tabla II muestra la lista de competencias deseables y/o funciones que debería llevar a cabo un técnico que trabajara con datos geográficos en la biblioteca. Esta lista está basada en el estudio de una serie de demandas de empleo para el perfil profesional identificado como geobibliotecario por parte de las universidades estadounidenses (Granell-Canut y Aguilar-Moreno, 2013). Algunas competencias se alinean perfectamente con las tareas tradicionales desempeñadas por un bibliotecario, adaptadas al contexto de datos geográficos: servicio de referencia (C1), alfabetización (C5), mantenimiento de la colección (C6), gestión de metadatos (C7), identificación de necesidades (C8), actividades de difusión (C9) y elaboración de recursos de información (C11). Otras son más afines a un especialista que llevara a cabo actividades de enlace (C2), investigación (C3), desarrollo de proyectos (C4), visualización de datos (C10) y representación institucional (C12) en materia de datos geográficos y SIG.

Esta agrupación de competencias no fue hecha explícita durante la encuesta, es decir, las bibliotecas encuestadas se limitaron a valorar en una escala de 1 (no importante) a 5 (muy importante) cada una de las competencias de la tabla II. No obstante, las respuestas obtenidas trazaron, a groso modo, los dos grupos de competencias apenas mencionados. Por una parte, las competencias con media igual o superior a 4 (C1, C6, C7, C9, C10, C11) se identifican con las competencias tradicionales que vienen desarrollándose en una biblioteca universitaria. Por otra parte, las competencias peor valoradas, con media menor de 4, son aquellas competencias relativas a tareas de gestión de datos geográficos (C4, C5, C8, C10) o que acarrean algún tipo de actividad de colaboración con departamentos e institutos universitarios (C2, C3, C12).

Tabla II. Competencias a desarrollar en la gestión de datos geoespaciales por los bibliotecarios

\begin{tabular}{|l|l|c|c|c|c|c|}
\hline & Descripción de la competencia/función & Min & Q1 & Media & Q4 & Max \\
\hline C1 & Servicio de referencia de datos geográficos & 2 & 3,75 & 4,07 & 5 & 5 \\
\hline C2 & $\begin{array}{l}\text { Actividades de enlace entre departamentos que lleven a } \\
\text { cabo actividades haciendo uso de datos geográficos }\end{array}$ & 2 & 3 & 3,79 & 5 & 5 \\
\hline C3 & $\begin{array}{l}\text { Participación en grupos de investigación internos sobre } \\
\text { datos geográficos }\end{array}$ & 1 & 3 & 3,40 & 4 & 5 \\
\hline C4 & Desarrollo de proyectos e iniciativas en datos geográficos & 1 & 3 & 3,40 & 4 & 5 \\
\hline C5 & $\begin{array}{l}\text { Alfabetización en datos geográficos y sistemas de infor- } \\
\text { mación geográfica }\end{array}$ & 2 & 3 & 3,82 & 4,25 & 5 \\
\hline C6 & $\begin{array}{l}\text { Mantenimiento de la colección: creación, organización, } \\
\text { análisis y preservación de datos geográficos }\end{array}$ & 3 & 3,75 & 4,14 & 5 & 5 \\
\hline C7 & Gestión de metadatos de los datos geográficos & 2 & 4 & 4,07 & 5 & 5 \\
\hline C8 & Identificación de necesidades en datos geográficos & 3 & 3 & 3,89 & 5 & 5 \\
\hline C9 & Actividades de difusión & 2 & 3 & 4,07 & 5 & 5 \\
\hline C10 & Promoción de "expertise" en visualización de datos geográficos & 2 & 3 & 3,46 & 4 & 5 \\
\hline C11 & $\begin{array}{l}\text { Elaboración de recursos de información sobre datos geográ- } \\
\text { ficos y sistemas de información geográfica }\end{array}$ & 2 & 3 & 4,07 & 5 & 5 \\
\hline C12 & $\begin{array}{l}\text { Participación en eventos como representante en materia de } \\
\text { datos geográficos del centro universitario }\end{array}$ & 1 & 2 & 3,25 & 4 & 5 \\
\hline
\end{tabular}

La tabla recoge estadísticas descriptivas (mínimo, primer cuartil, mediana, media, cuarto cuartil, máximo) de las valoraciones obtenidas $(\mathrm{N}=28)$. 
En las primeras experiencias de puesta en marcha de servicios de información geográfica (Janz, 1997 ) - principalmente en bibliotecas universitarias estadounidenses - se observa un gran interés por recoger información sobre software SIG, creación de directorios con enlaces a fuentes de datos, recursos, publicaciones, congresos, etc., incluso se habla de añadir a la colección aquellos datos que otros departamentos adquieran. También se considera a otros investigadores de la universidad como clientes del servicio, pero no como colaboradores para ponerlo en marcha (Longstreth, 1995). Estas mismas percepciones son las que los bibliotecarios universitarios españoles han transmitido en las respuestas sobre las competencias idóneas de un geobibliotecario (figura 4). Podríamos concluir que actualmente los bibliotecarios se encuentran más cómodos dentro de su rol tradicional, y que difícilmente se reconocen en el papel de formar parte de equipos de investigación o desarrollo de proyectos de SIG, aunque parece que se intuye tímidamente una posible aplicación de sus conocimientos a tareas de gestión de datos geográficos.

\section{CONCLUSIONES}

Respondiendo a los objetivos del presente trabajo, los resultados obtenidos confirman que existen trabas, si bien ocasionadas por motivos diferentes a los que se habían identificado en otros contextos universitarios, en cuanto a la puesta en marcha de servicios de gestión de datos geográficos. Como se explicó en la sección 3.2 no son los recursos económicos y humanos los responsables de que no se oferte este tipo de servicios por parte de las bibliotecas universitarias españolas, sino que sería el desconocimiento general en cuanto a la importancia de los datos geográficos para las universidades en el contexto de la gestión de datos de investigación. Del estudio también se desprende que, salvo honrosas excepciones, las bibliotecas universitarias españolas no estarían en disposición, con las herramientas de que disponen hoy en día, de facilitar servicios geográficos a la comunidad universitaria de forma satisfactoria. Si bien, a lo largo del artículo se ha visto que las respuestas dadas por las universidades españo-

Figura 4. Valoración de las competencias deseables para el perfil de geobibliotecario, N=28 (Bloque 4)

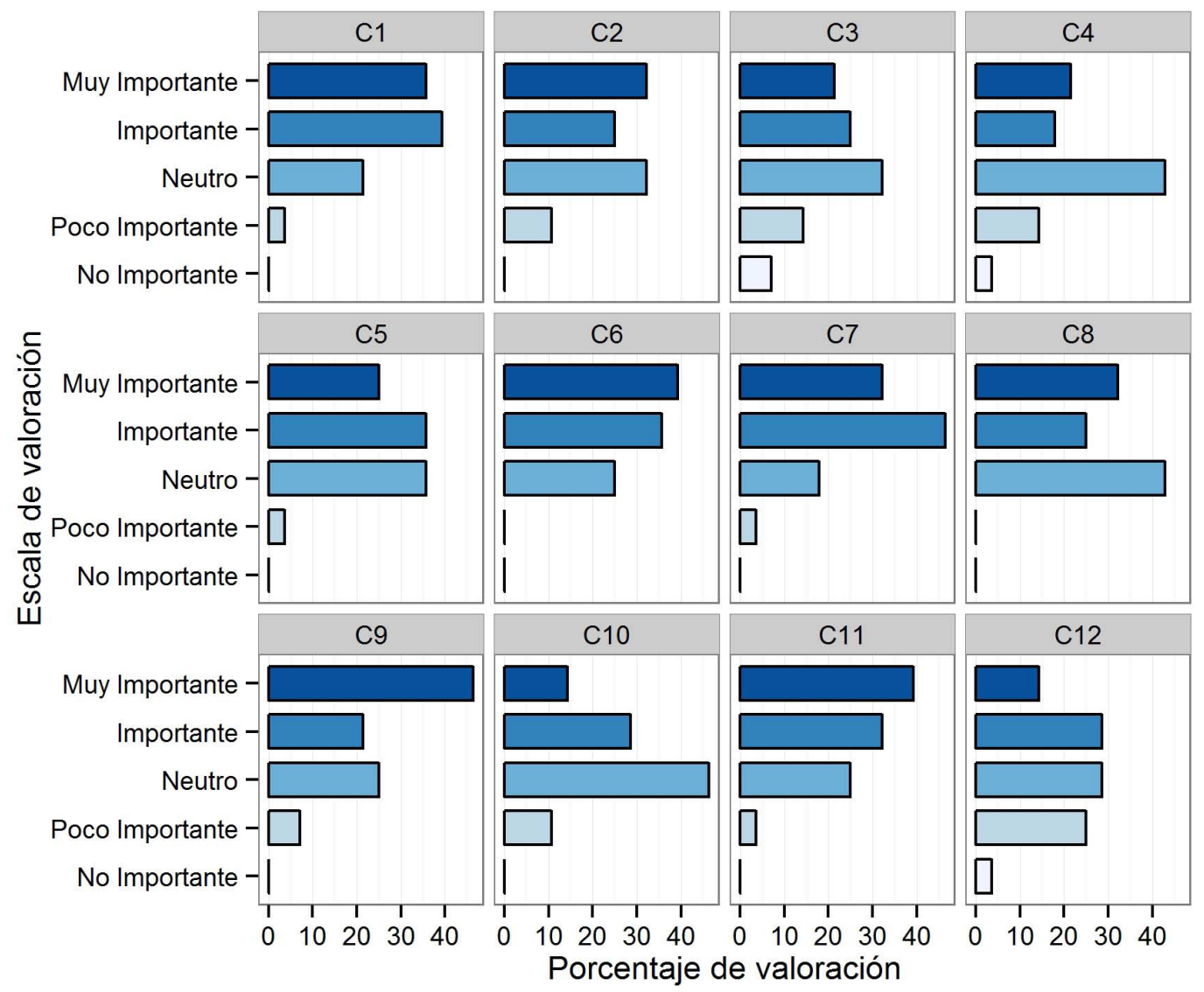


las difieren de las experiencias, desarrolladas y en funcionamiento, por parte de las universidades estadounidenses.

Varela-Pardo (2012) apunta que "/a misión de las bibliotecas académicas debe alinearse con la de la institución cuyas funciones son la educación y la investigación. Son necesarias capas profesionales y de práctica que contribuyan a mejorar la investigación y la enseñanza en todas las disciplinas". La alineación de objetivos implica necesariamente una estrecha colaboración entre las bibliotecas y los departamentos universitarios. Sin embargo, los resultados de la encuesta arrojan una situación totalmente opuesta, ya que las bibliotecas evidencian una falta de conocimiento sobre a qué nivel se está trabajando con datos geográficos en la comunidad académica a la que dan servicio. Esto supone una traba al establecimiento de colaboraciones entre bibliotecas y departamentos, y difícilmente hará de la biblioteca un centro neurálgico y de interacción en el campus universitario. Adicionalmente, sería deseable que las bibliotecas académicas recogieran y gestionaran datos geográficos no de forma puntual, atendiendo a directivas o reglamentos, sino como un procedimiento más dentro de sus tareas habituales alineado a estrategias institucionales conjuntas. Los problemas de preservación y mantenimiento que este tipo de datos presentan (Ariza López y otros, 2012) requieren abordar el problema desde una perspectiva multidisciplinar, donde equipos colaborativos desarrollen programas conjuntos de curación y preservación.

Sin embargo, no podemos afirmar categóricamente que la falta de entendimiento y desconocimiento mutuo entre la comunidad de investigadores y la biblioteca en cuanto a la gestión de datos geográficos se deba únicamente a la pasividad de estos dos actores. Hay otros factores internos y externos a la universidad que dificultan el establecimiento de colaboraciones y proyectos conjuntos entre bibliotecas y departamentos. Tal como apuntaba Varela-Pardo (2012), un impedimento bastante probable a nivel interno se deba al vacío estratégico institucional en cuanto a favorecer sinergias, proyectos conjuntos y el fomento de colaboraciones y relaciones entre departamentos y servicios de la universidad. Externamente, un factor determinante es la rápida aparición de tecnologías y aplicaciones (por ejemplo, los datos y tecnologías geoespaciales), que exigen la misma celeridad en la reflexión sobre el potencial impacto que puedan tener para la biblioteca.

Las bibliotecas en general, no reconocen la oportunidad de ampliar sus servicios y sentar unas bases sólidas para la gestión de datos de investigación, a través de la gestión de colecciones de datos geográficos. La íntima relación de los datos geográficos con datos abiertos y gestión de datos de investigación, los posiciona como pieza clave en las políticas universitarias de gestión de datos. A nadie se le escapa que la gestión de datos de investigación implica retos enormes, en cuanto a infraestructuras para el depósito de datos, necesidades de formación de personal especializado, controles de calidad de datos, licencias de compartición y uso de datos, entre otros. Pero también es cierto que representan un enorme nicho para las bibliotecas universitarias en un futuro no muy lejano, tal como augura el nuevo programa de investigación e innovación H2020 de la Comisión Europea (Comisión Europea, 2013).

El estudio también nos ha llevado a vislumbrar otra serie de conclusiones no previstas inicialmente en la preparación de la investigación, pero que consideramos suficientemente interesantes como para mencionarlas aquí.

En primer lugar, las bibliotecas españolas se encuentran en el mismo estado de incertidumbre en el que se encontraban las bibliotecas estadounidenses en los 90 cuando empezaron la puesta en marcha de servicios geográficos. En 2004 y 2005, cuando los servicios de Google Maps y Google Earth fueron lanzados, Fortin (2008) ya detectó un incremento de interés de datos geográficos por parte de los usuarios de la biblioteca. Si además añadimos la democratización de dispositivos que incorporan de forma habitual sistemas de geolocalización o GPS (Guan y otros, 2011), el interés por acceder a datos geográficos va a verse incrementado en los próximos años (Ariza López y otros, 2012). Las bibliotecas universitarias españolas necesitan hacer grandes esfuerzos en materia de gestión de datos geográficos si no quieren perder la estela de otras universidades en el ámbito internacional. En este sentido, es conveniente que las bibliotecas se sirvan de las experiencias previas desarrolladas en otros países si quieren lograr la máxima efectividad en los servicios geográficos que proporcionen.

En segundo lugar, la difusión de las políticas de datos abiertos y la apertura masiva de datos pueden provocar un interés por los datos geográficos. La publicación de la comunicación de la Comisión Europea - donde se cita específicamente a los datos geográficos - sobre la obligatoriedad de poner en abierto los resultados de investigación sufragados con fondos europeos (Comisión Europea, 2011), es una oportunidad más para que las bibliotecas académicas vean, en el cumplimiento de estas recomendaciones, una forma de ampliar su oferta de servicios. 


\section{NOTAS}

[1] Existen más bibliotecas universitarias en España, pero los autores consideran, que las que figuraban en el directorio REBIUN eran suficientemente representativas.

[2] El formulario de la encuesta y el listado de bibliotecas participantes se encuentra en https://github. com/cgranell/paper-geolibraries.

\section{BIBLIOGRAFÍA}

Aguilar-Moreno, E.; Granell-Canut, C. (2013). Sistemas de información geográfica para unidades de información. El Profesional de la Información, vol. 22(1), 80-86. http://dx.doi.org/10.3145/epi.2013.ene.11

Ariza López, F.J.; Ariza López, R.M.; Ureña Cámara, A. ; Cortes José, J. ; Ureña López, L.A. (2012). Preservación de la Información geográfica: perspectivas y situación de España. GeoFocus: Revista Internacional de Ciencia y Tecnología de la Información Geográfica, vol. 12, 171-200.

ARL (1999). The ARL Geographic Information Systems Literacy Project: a SPEC kit. Washington: Association of Research Libraries.

Blaschke, T.; Merschdorf, H. (2014). Geographic information science as a multidisciplinary and multiparadigmatic field. Cartography and Geographic Information Science, vol. 41(3), 196-213. http:// dx.doi.org/10.1080/15230406.2014.905755

Bosque González, I. del; Fernández Freire, C.; MartinForero Morente, L.; Pérez Asensio, E. (2012). Los Sistemas de información geográfica y la investigación en ciencias humanas y sociales. Madrid: CSIC. http://digital.csic.es/bitstream/10261/64940/1/ Los\%20SIG\%20y\%20la\%20Investigacion \%20 en $\% 20$ Ciencias $\% 20$ Humanas $\% 20 y \% 20$ Sociales.pdf

Cline, N.M.; Adler, P.S. (1995). SIG and research libraries: one perspective. Information Technology and Libraries, vol. 14(2), 111-115.

Comisión Europea (2011). Datos abiertos. Un motor para la innovación, el crecimiento y la gobernanza transparente COM (2011a) 882 final, Bruselas, 12.12.2011. http://eur-lex.europa.eu/LexUriServ/ LexUriServ.do? uri=COM: $2011: 0882$ :FIN : ES:PDF [Consulta: 2014-05-20].

Comisión Europea (2013). Guidelines on data management in Horizon 2020, 11/12/2013, http:// ec.europa.eu/research/participants/data/ref/ h2020/grants_manual/hi/oa_pilot/h2020-hi-oadata-mgt_en.pdf [Consulta: 2014-05-20].

Conway, E. ; Peppler, S. ; Garland, W. ; Hooper, D. ; Marelli, F. ; Liberti, L.; Piervitali, E.; Molch, K.; Glaves, H.: Badiali, L. (2013). Ensuring the long term impact of Earth science data through data curation and preservation. Information Standards Quarterly, vol. 25(3), 28-36. http://dx.doi.org/10.3789/isqv25no3.2013.05
[3] En aras de proporcionar transparencia y reproducibilidad del análisis realizado en esta investigación, tanto los datos obtenidos de la encuesta como el código del análisis están disponibles en línea y en abierto en el repositorio https://github. com/cgranell/paper-geolibraries. El documento Readme contiene indicaciones detalladas de todos los ficheros que aparecen en el repositorio.

Cullingworth, C. (2005). Implementation of Geographic Information Systems (GIS) into library and archival systems. Bulletin - Association of Canadian Map Libraries and Archives, vol. 122, 3-16.

Dodsworth, E. (2009). Developing geographical and geospatial skills in librarians. Bulletin - Association of Canadian Map Libraries and Archives, vol. 135, 10-14.

Florance, P (2006). GIS collection development within an academic library. Library Trends, vol. 55(2), 222-235. http://dx.doi.org/10.1353/lib.2006.0057

Fortin, M. (2008). Map and GIS librarianship in a Google Earth world. Bulletin - Association of Canadian Map Libraries and Archives, vol. 133, 3-10.

Gabaldon, C.; Repplinger, J. (2006). GIS and the academic library: A survey of libraries offering GIS services in two consortia. Issues in Science and Technology Librarianship, fall. http://dx.doi. org/10.5062/F4QJ7F8R

Goldberg, D.; Olivares, M.; Li, Z.; Klein, A.G. (2014). Maps \& GIS data libraries in the era of big data and cloud computing. Journal of Map and Geography Libraries, vol. 10, 100-122. http://dx.doi.org/10.1 080/15420353.2014.893944

Gould, M. (2013). Spatial Thinking and Smart Campus Projects en: 2013 Association of American Geographers (AAG) Annual Meeting. Los Angeles California.

Granell-Canut, C.; Aguilar-Moreno, E. (2013). Se busca geobibliotecario: Los datos geográficos entran en la biblioteca. El Profesional de la Información, vol. 22(6), 569-575. http://dx.doi.org/10.3145/ epi.2013.nov.10

Guan, W.W.; Burns, B.; Finkelstein, J.L.; Blosson, J.C. (2011). Enabling geographic research across disciplines: building and Institutional infrastructure for geographic analysis at Harvard University. Journal of Map and Geography Libraries, vol. 7, 36-60. http://dx.doi.org/10.1080/15420353.2011.534688

Hernández-Pérez, T.; García-Moreno, M.A. (2013). Datos abiertos y repositorios de datos: nuevo reto para los bibliotecarios. El Profesional de la Información, vol. 22(3), 259-263. http://dx.doi. org/10.3145/epi.2013.may.10 
Herrera-Morillas, J. L. (2013). La gestión de la colección en las bibliotecas universitarias españolas. Planes y normativas: la selección. Revista Española de Documentación Científica, vol. 36(4), e021. http:// dx.doi.org/10.3989/redc.2013.4.986

Janz, Ronald C. (1997). Geographical information system at Princeton University: evaluation and exploration of a new library service. Collection Building, vol. 16 (3), 125-136. http://dx.doi. org/10.1108/01604959710172413

Johnson, A.; Phoenix, M. (2003). GIS across the campus. ArcUser, Abril Junio 2003, 16-17. http:// www.esri.com/news/arcuser/0403/focus1of2.html

Kelly, L. (2013). Maps, libraries and the "GIS Librarian": an informal review of international cartographic libraries. South African Journal of Geomatics, vol. 2 (2), 163-174. http://www.sajg.org.za/index.php/ sajg/article/view/77 [Consultado: 2014-05-15]

Kinikin, J.; Hench, K. (2005a). Survey of GIS implementation and use within a smaller academic libraries. Issues in Science and Technology Librarianship, spring. http://dx.doi.org/10.5062/ F4SX6B56

Kinikin, J.; Hench, K. (2005b). Follow-up survey of GIS at smaller academic libraries. Issues in Science and Technology Librarianship, summer. http:// dx.doi.org/10.5062/F4S46PWT

Longstreth, K. (1995). GIS collection development, staffing, and training. Journal of Academic Librarianship, vol. 21 (4), 267-274. http://dx.doi. org/10.1016/0099-1333(95)90006-3

Lorite, M. (2011). Los recursos cartográficos en las bibliotecas universitarias españolas. En: Actas de las primeras jornadas virtuales Iberoamericanas de Ciencias de la Información y la Documentación. 10-30 Octubre 2011. http://www.congresosweb. info/index.php?option $=$ com mtree\&task $=$ att download\&link_id $=63 \& c f \_i d=24$

MAGERT: Map, GIS and cataloguing/metadata librarian core competencies. (2008). ALA. http://www. ala.org/magirt/sites/ala.org.magirt/files/content/ publicationsab/MAGERTCoreComp2008_rev2012. pdf [Consultado: 2014-05-15]
Martindale, J. (2004). Geographic Information Systems Librarianship: Suggestions for Entry-Level Academic Professionals. The Journal of Academic Librarianship, vol. 30(1), 67-72. http://dx.doi.org/10.1016/j. jal.2003.11.008

Pérez Pulido, M. (1999). Consideraciones acerca de la utilización de sistemas de Información Geográfica en la planificación de servicios bibliotecarios. En: 7 Jornades Catalanes de Documentacio. pp. 271277. http://www.cobdc.org/jornades/7JCD/38.pdf [Consultado: 2014-05-15].

Scaramozzino, J.; White, R.; Essic, J.; Fullingotn, L.A.; Mistry, H.; Henley, A.; Olivares, M. (2014). Map room to data and GIS services: five university libraries evolving to meet campus needs and changing technologies. Journal of Map and Geography Libraries, vol. 10, 6-47. http://dx.doi.org/10.1080 /15420353.2014.893943

Vardakosta, I.; Kapidakis, S. (2011a). Geographical collections in Greek academic libraries: Current situation and perspectives. En International Conference on Integrated Information, Kos (GR), September 29 - October 3, 2011. Available at: http://hdl.handle.net/10760/16169

Vardakosta, I.; Kapidakis, S. (2011b). Geospatial collections development policies in academic libraries: A worldwide research. En 17th European Colloquium on Quantitative and Theoretical Geography (ECQTG2011), Athens (GR), September 2-5. http://hdl. handle.net/10760/16096

Vardakosta, I.; Kapidakis, S. (2012). Geospatial data in library collections. ACM International conference proceedings series. Art 33. http://bit.ly/1cC5r2I

Varela-Pardo, C; Baiget, T. (2012). El futuro de las bibliotecas académicas: incertidumbres, oportunidades y retos. Investigación Bibliotecológica, vol. 26 (56), 115-135. http://bit.ly/1cC5r2I

Weimer, K.H.; Reehling, P. (2006). A new model of Geographic Information Librarianship: description, curriculum and program proposal. Journal of Education for Library and Information Science, vol. 47 (4), 291-302. http://dx.doi.org/10.2307/40323822 